\title{
Relation between dust and radio luminosity in optically selected early type galaxies
}

\author{
D. Krajnović^ and W. Jaffe
}

\begin{abstract}
Sterrewacht Leiden, Postbus 9513, 2300 RA Leiden, The Netherlands
Received 21 February 2002 / Accepted 7 May 2002

Abstract. We have surveyed an optical/IR selected sample of nearby E/S0 galaxies with and without nuclear dust structures with the VLA at $3.6 \mathrm{~cm}$ to a sensitivity of $100 \mu \mathrm{Jy}$. We can construct a Radio Luminosity Function (RLF) of these galaxies to $\sim 10^{19} \mathrm{~W} \mathrm{~Hz}^{-1}$ and find that $\sim 50 \%$ of these galaxies have AGNs at this level. The space density of these AGNs equals that of starburst galaxies at this luminosity. Several dust-free galaxies have low luminosity radio cores, and their RLF is not significantly less than that of the dusty galaxies.
\end{abstract}

Key words. galaxies: elliptical and lenticular, $\mathrm{cD}$ - galaxies: luminosity function, mass function - galaxies: nuclei

\section{Introduction}

Research conducted during the last decade gave a new view of nearby elliptical galaxies previously considered as old, uniform systems with little gas or dust. Images from the Hubble Space Telescope (HST) have shown that many early-type galaxies have a large amount of dust $\left(10^{3}-10^{7} M_{\odot}\right)$, either in the form of a nuclear disk or in the more diverse form of filaments. Among different studies there is a large variation in the detection rates which may be due to the different methods, resolutions, and sensitivities of the observations (Sadler \& Gerhard 1985, 40\%; Goudfrooij et al. 1994, 41\%; van Dokkum \& Franx 1995, 48\%; Ferrari et al. 1999, 75\%; Tomita et al. 2000, 56\%; Rest et al. 2001, 43\%; Tran et al. 2001 (IRAS bright sample), $78 \%$ ), but the general conclusion is that dust is common in nearby ellipticals.

Establishing the presence of dust in nearby early-type galaxies is only the first step towards determining the role of dust in these systems. It is already a well-known fact that radioloud ellipticals often have large amounts of dust but there are some open questions, especially for the radio-weak sources. Verdoes Kleijn et al. (1999) found that the incidence of dust in radio-loud early type galaxies is $89 \%$ while Tran et al. (2001) has a value of $43 \%$ for the occurrence of dust in their snapshot sample of relatively radio-quiet nearby early-type galaxies (for a description of the sample see Sect. 2). In the same sample, $66 \%$ of dusty galaxies have NRAO VLA Sky Survey (NVSS) $1.4 \mathrm{GHz}$ flux detections (Condon et al. 1998), while only $8 \%$ of galaxies without dust are listed as radio sources.

These results raise a question: how important is the presence of dust for radio emission in the nuclei of ellipticals?

Send offprint requests to: D. Krajnović, e-mail: davor@strw.leidenuniv.nl

* On leave of absence from Institute Rudjer Boskovic, Bijanicka cesta 54, 10000 Zagreb, Croatia.
Plausibly, dust indicates the presence of gas, and gas is necessary to fuel the activity of a central massive black hole (BH). However, this line of reasoning is highly incomplete. Gas may be present without dust. Dust may be present but not visually detectable (Goudfrooij \& de Jong 1995). Dust and gas that have fed a BH in the past may not be observable at the time when the nuclear activity is observed. These arguments justify a careful study of the relation between dust and nuclear radio emission to determine the relevance of radio luminosity, dust morphology and other effects.

There are two approaches to the study of extragalactic radio sources. The first one is based on catalogs of discrete radio sources followed by an analysis of the optical counterparts. The second involves searching for radio emission from optically chosen objects. The first approach (e.g. de Koff et al. 2000) is relatively efficient in finding radio galaxies, but emphasizes powerful radio sources and may not provide a good countersample of radio-quiet galaxies. The second approach conversely emphasizes weak radio sources (e.g. Sadler et al. 1989; Wrobel 1991; Wrobel \& Heeschen 1991; Sadler et al. 2002).

Both types of radio surveys are important. Here we have chosen the second method primarily so that the optical selection of the sample, including Hubble type and especially dust content, is not biased by a priori selection for radio emission or other "interesting" properties of the galaxies. The survey objects are selected on their optical/IR properties only and then observed with the VLA with the purpose of establishing the presence of nuclear AGNs. We compare our dusty and nondusty parts of the sample to investigate the importance of dust (as a fuel reservoir) for the existence of nuclear activity.

In Sect. 2 we present the sample and discuss the observations and the data reduction. In Sect. 3 we present the results of our study. They are followed with a discussion in Sect. 4. 
Sect. 5 brings a discussion on correlation of dust with radio emission. The conclusions are given in Sect. 6.

\section{Observations}

\subsection{Sample}

Our sample is compiled from two different samples described by Rest et al. (2001) and Tran et al. (2001). The first sample was created by selecting E/S0 galaxies on their optical properties only from the Lyon/Meudon Extragalactic Database (LEDA). A randomly selected subset of 68 galaxies from this sample was observed with HST using WFPC2 in snapshot mode and thus this sample is referred to as the "snapshot" sample. An additional sample of galaxies was assembled from archival HST images of nearby E/S0 galaxies selected for their $100 \mu \mathrm{m}$ IRAS emission as these were likely to contain large amount of dust (Tran et al. 2001). This sample is referred to as the "IRAS sample". From these two samples, we have selected 36 objects (18 from each) according to their optical/IR properties, with no regard to radio properties. The reason for this selection was to avoid biasing in picking a priori "interesting" objects and objects with strong radio fluxes. Galaxies in our sample are nearby ellipticals and lenticulars (E/S0), $c z<3200 \mathrm{~km} \mathrm{~s}^{-1}$, at galactic latitude exceeding $20^{\circ}$ to minimize Galactic extinction, and with absolute $V$-band magnitude less then -17 . Because of their optical/IR selection they tend to have low radio powers.

The global properties of galaxies in our sample are listed in Table 1 of Rest et al. (2001) and in Table 7 of Tran et al. (2001). In the list of 36 galaxies, 18 of them were chosen because they have dust in the form of disks or filaments. The other 18 nondusty galaxies were selected to match dusty galaxies in optical properties, redshift, magnitude, and IRAS flux. However, after the initial selection, more detailed studies (Rest et al. 2001, Tran et al. 2001) showed that 6 of the "non-dusty" galaxies showed faint dust structures and have here been included in the "dust" class. We used $H=80 \mathrm{~km} \mathrm{~s}^{-1} \mathrm{Mpc}^{-1}$ to be consistent with the papers defining the samples.

\subsection{Data acquisition and reduction}

The observations were undertaken with the VLA in C configuration at $3.6 \mathrm{~cm}$ wavelength. All sources were observed at two frequencies in the $8 \mathrm{GHz} X$-band $(8.4351$ and $8.4851 \mathrm{GHz})$ with a bandwidth of $50 \mathrm{MHz}$ for each frequency. We observed 68 sources in total, 36 galaxies and 32 calibrators. Each galaxy was observed for $15 \mathrm{~min}$ while calibrators were observed for 130 seconds. Most of the calibrators had position code A (positional accuracy $<0$ ' 002$)$, but four calibrators had B $\left(00^{\prime} 002-\right.$ $\left.0{ }^{\prime} 01\right)$ and three had $\mathrm{C}\left(0{ }^{\prime} 01-0{ }^{\prime} 15\right)$ as is indicated on the calibrator web page of the VLA. The radio positions of the detected sources are limited by this positional accuracy of the calibrators, as well as by the accuracy of the Gaussian fit to the source brightness distribution, which is dependent on the signal-to-noise ratios. Taking this in account the overall accuracy is about 50 mas for mJy sources and about 100 mas for $100 \mu \mathrm{Jy}$ sources. The observations were taken on March 13, 2000.
We used the Astronomical Image Processing System (AIPS) to reduce the data using the standard procedures from the AIPS cookbook. After initial calibration, the data were imaged using the task IMGR. The data were self-calibrated in phases to improve the image dynamic range, using a model derived from the same data. In some cases amplitude selfcalibration was performed on the data to improve the final images. For our astrometric purpose, the positions of the sources were extracted before self-calibration so that phase information was preserved. All the images were examined using the tasks JMFIT and IMSTAT.

\section{Results}

Twenty galaxies in our sample of 36 were detected as radio sources. Three detected sources (associated with NGC 2986, NGC 3610, NGC 4125) cannot be matched with the central regions of the galaxies and there are no visible counterparts on the available HST pictures, hence they are most likely background sources. The radio sources lay far from the nuclei (about 2'.67 for NGC 2986, 3'.84 for NGC 3160, and 1'.44 for NGC 4125). Although the fluxes and positions of these sources are listed in Table 1 (with asterisks) we treat them as non-detections of central AGNs in the surveyed galaxies. This leaves 17 AGN detections in 36 galaxies (47\% detection rate). The smallest signal to noise ratio $(S N R)$ is about $10 \sigma$ with a survey average rms $\sigma=2.8 \times 10^{-5} \mathrm{Jy} /$ beam. For non-detected sources we calculated the $4 \sigma$ upper limits on detection, thus, the detection limit of our survey is about $0.1 \mathrm{mJy}$. Radio properties of the sample are given in Table 1. By comparison, the detection limit of the NVSS (Condon et al. 1998) used by Tran et al. (2001) to discuss radio properties of our sample is $\sim 3 \mathrm{mJy}$, a factor of 30 higher.

Most of the detections are point-like, unresolved structures. NGC 5322 is the only galaxy with noticeable jet-like structure. Typical detected sources are on the level of a few mJy; the weakest detections were $\sim 200 \mu \mathrm{Jy}$. Of the 36 galaxies in the sample, 24 galaxies show disk or filamentary dust structure and $13(54 \%)$ of them are detected as radio sources. Twelve show no dust of which four (33\%) are detected.

\section{Discussion}

\subsection{Nature of detected radio sources}

Most of the detections are unresolved radio sources easily associated with the central 1".5 on the HST image. At $25 \mathrm{Mpc}$, the mean distance of the galaxies in the sample, $1^{\prime \prime}$ is about $120 \mathrm{pc}$. Thus the emission is clearly (near) nuclear, but not necessarily of AGN origin. Since the sources are weak (radio power ranges from $10^{19} \mathrm{~W} \mathrm{~Hz}^{-1}$ to $10^{21} \mathrm{~W} \mathrm{~Hz}^{-1}$ with a few higher exceptions) there is a possibility that they arise from a non-AGN mechanism, e.g. nuclear starbursts. Since we are interested in the AGN/dust connection we wish to exclude this possibility. We argue here that the dominant source of radio emission in our detections is a non-thermal mechanism similar to that which operates in more powerful radio sources. 
Table 1. Radio properties of the galaxies.

\begin{tabular}{|c|c|c|c|c|c|c|c|c|}
\hline $\begin{array}{c}\text { name } \\
(1)\end{array}$ & $\begin{array}{l}\text { dust } \\
\text { (2) }\end{array}$ & $\begin{array}{l}D \\
(3)\end{array}$ & $\begin{array}{c}\text { peak flux } \\
\text { (4) }\end{array}$ & $\begin{array}{l}\text { RA } \\
(5)\end{array}$ & $\begin{array}{l}\text { Dec } \\
(6)\end{array}$ & $\begin{array}{c}L \\
(7)\end{array}$ & $\begin{array}{c}\delta \\
(8)\end{array}$ & $\begin{array}{c}\text { NVSS flux } \\
\text { (9) }\end{array}$ \\
\hline ngc 1400 & 2 & $25.4^{\mathrm{P}}$ & $2.092 \pm 0.02$ & 033930.815 & -184117.42 & $1.61 \pm 0.02$ & 1.80 & $2.5 \pm 0.5$ \\
\hline ngc 1439 & 4 & $20.9^{\mathrm{T}}$ & $<0.1$ & & & & & \\
\hline ngc 2549 & 0 & $15.7^{\mathrm{R}}$ & $<0.1$ & & & & & \\
\hline ngc 2592 & 4 & $25.5^{\mathrm{R}}$ & $0.41 \pm 0.02$ & 082708.040 & 255813.00 & $0.32 \pm 0.01$ & 0.65 & \\
\hline ngc 2699 & 4 & $21.8^{\mathrm{R}}$ & $<0.1$ & & & & & \\
\hline ngc 2768 & 4 & $16.7^{\mathrm{T}}$ & $10.71 \pm 0.02$ & 091137.418 & 600214.84 & $3.59 \pm 0.01$ & 0.54 & $14.5 \pm 0.6$ \\
\hline ngc 2778 & 0 & $25.4^{\mathrm{T}}$ & $<0.1$ & & & & & \\
\hline ngc 2974 & 3 & $25.9^{\mathrm{T}}$ & $5.22 \pm 0.02$ & 094233.310 & -034157.09 & $4.19 \pm 0.02$ & 0.93 & $10.4 \pm 0.5$ \\
\hline ngc $2986^{*}$ & 0 & $22.3^{\mathrm{T}}$ & $8.40 \pm 0.03$ & 094427.256 & -211611.23 & & 160.14 & \\
\hline ngc 3078 & 4 & $29.0^{\mathrm{R}}$ & $124.95 \pm 0.04$ & 095824.630 & -265536.09 & $125.73 \pm 0.04$ & 1.45 & $279 \pm 8$ \\
\hline ESO 437-15 & 3 & $32.3^{\mathrm{R}}$ & $1.76 \pm 0.04$ & 103658.100 & -281034.70 & $2.20 \pm 0.05$ & 0.80 & $3.2 \pm 0.6$ \\
\hline ngc 3156 & 2 & $14.0^{\mathrm{T}}$ & $<0.1$ & & & & & \\
\hline ngc 3226 & 3 & $17.3^{\mathrm{R}}$ & $7.29 \pm 0.05$ & 102327.005 & 195354.75 & $2.61 \pm 0.02$ & 0.97 & \\
\hline ngc 3348 & 0 & $38.5^{\mathrm{R}}$ & $1.66 \pm 0.02$ & 104710.000 & 725022.71 & $2.94 \pm 0.04$ & 1.36 & $7.8 \pm 0.5$ \\
\hline ngc 3377 & 1 & $9.1^{\mathrm{T}}$ & $<0.1$ & & & & & \\
\hline ESO 378-20 & 0 & $35.6^{\mathrm{R}}$ & $<0.1$ & & & & & \\
\hline ngc 3595 & 0 & $30.4^{\mathrm{R}}$ & $0.22 \pm 0.01$ & 111525.180 & 472650.60 & $0.24 \pm 0.01$ & 3.87 & \\
\hline $\operatorname{ngc} 3610^{*}$ & 3 & $26.8^{\mathrm{R}}$ & $1.17 \pm 0.03$ & 111820.700 & 584938.11 & & 230.78 & \\
\hline $\operatorname{ngc} 4125^{*}$ & 4 & $20.1^{\mathrm{R}}$ & $1.23 \pm 0.02$ & 120804.180 & 650941.32 & & 86.29 & \\
\hline ngc 4233 & 4 & $29.6^{\mathrm{R}}$ & $2.52 \pm 0.01$ & 121707.679 & 073727.33 & $2.64 \pm 0.01$ & 1.02 & $2.9 \pm 0.5$ \\
\hline $\operatorname{ngc} 4365$ & 0 & $15.7^{\mathrm{R}}$ & $<0.1$ & & & & & \\
\hline ngc 4406 & 4 & $17.0^{\mathrm{V}}$ & $0.59 \pm 0.02$ & 122611.770 & 125646.40 & $0.204 \pm 0.07$ & 1.37 & \\
\hline $\operatorname{ngc} 4476$ & 3 & $24.7^{\mathrm{T}}$ & $<0.1$ & & & & & \\
\hline ngc 4494 & 4 & $17.8^{\mathrm{R}}$ & $0.27 \pm 0.01$ & 123124.030 & 254630.01 & $0.10 \pm 0.01$ & 2.00 & \\
\hline ngc 4552 & 4 & $17.0^{\mathrm{V}}$ & $93.40 \pm 0.02$ & 123539.805 & 123322.78 & $32.30 \pm 0.01$ & 0.35 & $100 \pm 3$ \\
\hline ngc 4697 & 4 & $15.5^{\mathrm{T}}$ & $<0.1$ & & & & & \\
\hline ngc 4742 & 3 & $15.9^{\mathrm{T}}$ & $<0.1$ & & & & & \\
\hline ngc 5198 & 0 & $34.1^{\mathrm{R}}$ & $0.83 \pm 0.02$ & 133011.390 & 464014.80 & $1.15 \pm 0.03$ & 1.16 & $3.6 \pm 0.4$ \\
\hline ngc 5322 & 4 & $23.9^{\mathrm{T}}$ & $13.60 \pm 0.02$ & 134915.269 & 601125.92 & $9.33 \pm 0.01$ & 1.08 & $64 \pm 2$ \\
\hline ngc 5557 & 0 & $42.5^{\mathrm{R}}$ & $<0.1$ & & & & & \\
\hline ngc 5576 & 0 & $19.1^{\mathrm{R}}$ & $<0.1$ & & & & & \\
\hline ngc 5812 & 4 & $24.6^{\mathrm{R}}$ & $<0.1$ & & & & & \\
\hline ngc 5813 & 4 & $24.6^{\mathrm{R}}$ & $2.95 \pm 0.02$ & 150111.234 & 014207.10 & $2.14 \pm 0.01$ & 0.72 & $12.3 \pm 0.7$ \\
\hline ngc 5845 & 4 & $18.1^{\mathrm{T}}$ & $<0.1$ & & & & & \\
\hline ngc 5982 & 0 & $39.3^{\mathrm{R}}$ & $<0.1$ & & & & & \\
\hline $\operatorname{ngc} 6278$ & 0 & $37.1^{\mathrm{R}}$ & $1.06 \pm 0.01$ & 170050.325 & 230039.73 & $1.75 \pm 0.02$ & 0.62 & \\
\hline
\end{tabular}

Column 1: name of the galaxy, sources with a star are far from the nuclear region of the corresponding galaxies and were treated as non detections; Col. 2 : level of dust: $0=$ no dust, $1=$ filamentary low, $2=$ filamentary medium, $3=$ filamentary high, $4=$ dusty disk (Tran et al. 2001); Col. 3: distance in Mpc from (P) - Perrett et al. 1997, (T) - Tran et al. 2001, (R) - Rest et al. 2001, (V) - Virgo galaxies, assumed to be at distance of $17 \mathrm{Mpc}$; Col. 4: flux at 3.6cm in mJy, or $4 \sigma$ upper limits for nondetections; Cols. 5 and 6: radio position (h, m, s) and (deg, arcmin, arcsec) from our maps (J2000); Col. 7: luminosity in $10^{20} \mathrm{WHz}^{-1}$; Col. 8: offset in arcseconds, between the $3.6 \mathrm{~cm}$ radio position and the position of the galaxy optical nucleus on HST images (Tran et al. 2001, Rest et al. 2001); Col. 9: peak flux from NVSS survey (Condon et al. 1998).

There are several radio and infrared criteria that can be used to distinguish between emission from starburst and AGN galaxies: (i) radio morphology, (ii) far-infrared to radio flux-density parameter $u \equiv \log \left(S_{60 \mu \mathrm{m}} / S_{1.4 \mathrm{GHz}}\right)$, (iii) infrared spectral index $\alpha_{\mathrm{IR}} \equiv \log \left(S_{60 \mu \mathrm{m}} / S_{25 \mu \mathrm{m}}\right) / \log (60 / 25)$
(Condon \& Broderick 1988, 1991; Condon et al. 1991; Condon et al. 1991), and (iv) the steepness of the radio spectra. Radio morphology implies coherent radio jets and radio lobes that may lie well outside the optical galaxy. Starburst galaxies usually have $u \geq 1.6$, and $\alpha_{\mathrm{IR}} \geq+1.25$. Steepness of the 
radio spectrum is also used as a criterion since optically thick AGN cores usually have flat spectra, while the dominant emission from star-forming regions (supernova remnants, and cosmic rays diffusing from them) have steep spectra. Nearly all spirals and unclassifiable objects (e.g. mergers) have steep spectra, while flat spectra and other AGN characteristics (radio morphology, $u \leq 1.6$, and $\alpha_{\mathrm{IR}} \leq+1.25$ ) are associated with ellipticals (Sadler et al. 1989; Condon 1991).

All detected galaxies in our sample have low-luminosity unresolved sources in the innermost central regions. Although the sources are certainly nuclear in origin (suggesting AGN activity) any radio classification according to radio morphology is not possible (except in the clear case of a jet in NGC 5233). Half of the galaxies were picked based on their large-scale dust and infrared properties from the IRAS survey. This means that those galaxies are going to have larger $\alpha_{I R}$ indices, which would mark them as starburst, although they still might have nuclear AGN which are the subject under discussion. Using the large scale IR emission to determine the nature of the nuclear radio emission does not seem to be a very good discriminator between SBs and AGNs. However, most of our objects have measured nuclear $\mathrm{H} \alpha$ fluxes or upper limits (Tran, private communication), and standard calculations (Osterbrock 1989) indicate that the free-free fluxes from these regions would be below $3 \mu \mathrm{Jy}$, which is about two to three orders of magnitude smaller than our observed fluxes. Other evidence that we are dealing with non-thermal radiation comes from the flatness of the spectra in our sample. Eleven of the galaxies were detected before in the NVSS (Condon et al. 1998) and comparing the fluxes at our frequency $(8.45 \mathrm{GHz})$ and the frequency of the NVSS $(1.4 \mathrm{GHz})$ it is clear that most of the detected galaxies have flat spectra (Table 1).

Previous studies (Phillips et al. 1986; Sadler et al. 1989) have shown that HII regions in early type galaxies are not likely to contribute to the radio galaxy population above $10^{19} \mathrm{~W} \mathrm{~Hz}^{-1}$. Keeping in mind that all galaxies in our sample are Es and S0s, that emission is confined to nuclei of the host galaxies, and that the sources have flat spectra, we can assume that the dominant radio component in our case is synchrotron emission from an active nucleus producing low-luminosity counterpart of more distant, luminous AGNs.

\subsection{Radio luminosity function}

The size of our sample is too small and too limited in radio luminosity range to construct a complete local radio luminosity function (RLF) of early-type galaxies. In any case, the sample was not constructed for that purpose. Still, we can make a useful estimate of the low luminosity end of the local RLF in order to see how it corresponds with previously found local RLFs and offer an estimate of the behavior of RLF at low luminosities. For this purpose we define the fractional luminosity function (Auriemma et al. 1977):

$F_{i}(L, z)=\rho_{i}(L, z) / \varphi_{i}(z)$,

where $\varphi_{i}(z)$ is the volume density of objects of type $i$ at the redshift $z$, and $\rho_{i}(L, z)$ is the density of sources associated with optical objects of type $i$ with the given radio luminosity $L$ and at

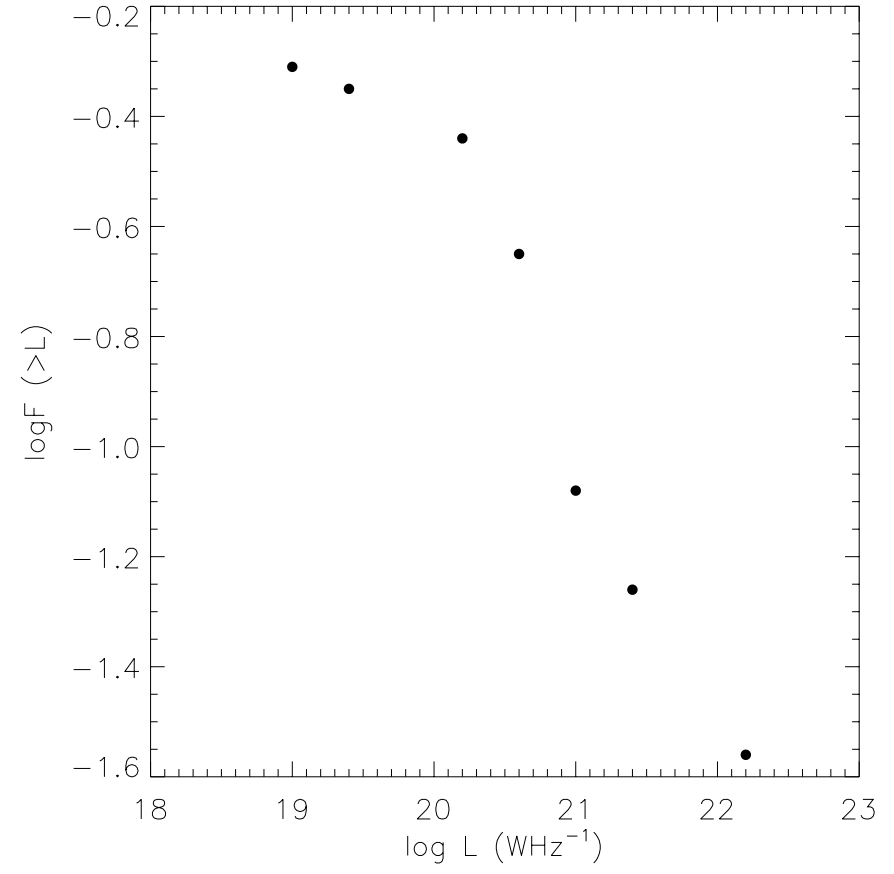

Fig. 1. The integral luminosity function derived from 17 detected sources out of a total of 36 . The dots represent a crude LF calculated from the detections as the integral of a series of delta functions. A detection at $L_{i}$ contributes $1 / N_{\mathrm{d}}\left(L_{i}\right)$ where the denominator is the number surveyed galaxies detectable at $L_{i}$. The steep rise flattens off below of $10^{20} \mathrm{~W} \mathrm{~Hz}^{-1}$. The error bars are not plotted since the bins in the integral RLF are not independent.

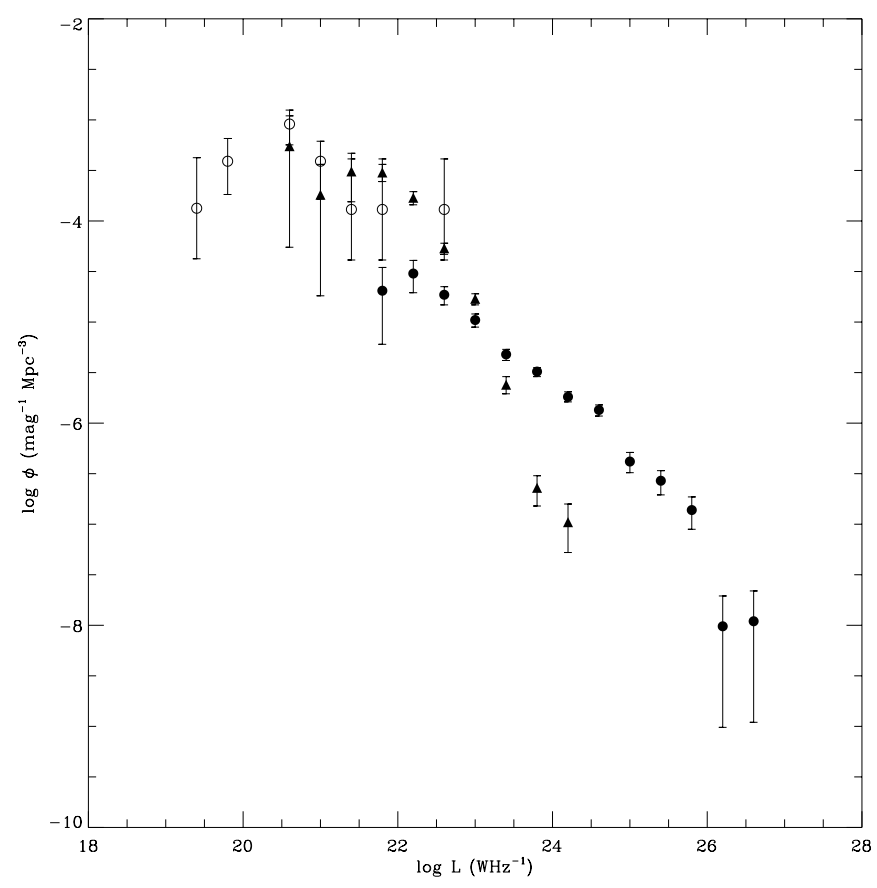

Fig. 2. Comparison of AGN and starburst (SB) local radio luminosity function. Filled symbols (circles: AGN, triangles: SB) are data from Sadler et al. (2002) and Condon (1991), while open circles are our data. The local density of AGN rises continuously at low luminosities, reaching the value of $\mathrm{SB}$, suggesting that AGNs are as common as $\mathrm{SB}$ in local universe. It is possible that at this low luminosity level both processes are present in galaxies, but in some galaxies one of the engines is stronger. 
the given redshift $z$. The fraction of all elliptical galaxies with luminosity at a given frequency between $L$ and $L+\mathrm{d} L$ at the redshift $z$, of optical magnitude $M$ is given then by $F_{E, M}(L, z) \mathrm{d} L$. In order to estimate the "bivariate" RLF defined in this way we can calculate the fractional detection $f_{i j}=n_{i j} / N_{i j}$, where $n\left(L_{i}, M_{j}\right)$ is the number of actually detected galaxies within the optical magnitude range $M_{j} \pm 0.5$ and radio luminosity interval $\log L_{i} \pm 0.2$, while $N\left(L_{i}, M_{j}\right)$ is the number of galaxies in the sample which could have been detected if their optical magnitude and radio luminosity were in the given interval. In our case we did not bin in optical magnitude but only in radio power since the sample is limited. Our first estimate of the RLF is then given by $f_{\mathrm{L}}=n_{\mathrm{L}} / N_{\mathrm{L}}$ and it is shown in Fig. 1 in integral form, $F(>L)$. As it can be seen from the Figure 1, the integral RLF of nearby ellipticals rises steeply with decreasing of the radio luminosity and only at the lowest intensities $\left(10^{19} \mathrm{~W} \mathrm{~Hz}^{-1}\right)$ levels off at a point where $\sim 50 \%$ of all E/S0 galaxies show activity.

Previous RLFs (Auriemma et al. 1977; Sadler et al. 1989; Condon 1991; Sadler et al. 2002) of nearby ellipticals with an AGN signature were made for galaxies with radio luminosities higher than $10^{21}-10^{22} \mathrm{~W} \mathrm{~Hz}^{-1}$. The more recent studies considered also starburst galaxies. While AGN were found in ellipticals, starbursts inhabited spirals. These different distributions had different RLFs and often starburst RLFs extended to the level of $10^{20} \mathrm{~W} \mathrm{~Hz}^{-1}$. With our low luminosity data, we are able to extend the existing RLFs of AGN down to $10^{19} \mathrm{~W} \mathrm{~Hz}^{-1}$ and can construct an exclusively AGN RLF.

We compare our data with two studies (Sadler et al. 1989; Condon 1991) in Fig. 2 (AGNs and starbursts plotted). We have converted our differential data from $F(L)$ to a spatial density $\phi$ (number of sources per $\mathrm{Mpc}^{3}$ per 0.4 in $\log L$ ) using the value for spatial density of early type galaxies, from Sadler et al. (1989), which is $10^{-2.33} \mathrm{mag}^{-1} \mathrm{Mpc}^{-3}$. Gratifyingly our data agrees quite well with the previous data in the region of overlap. Together these data confirm the flattening of the RLF for AGNs below $\sim 10^{20} \mathrm{~W} \mathrm{~Hz}^{-1}$. It is also interesting that the space density of low luminosity AGN is very similar to starbursts galaxies of the same luminosities. The RLFs of the two distributions are basically overlapping in this luminosity range.

\section{Correlation of dust with radio emission}

\subsection{Crude statistics}

The HST pictures of the galaxies in the original sample (Tran et al. 2001) confirm that dust is very common in ellipticals. There are two different morphologies in which dust appears in the galaxies from our sample: disky and filamentary. We have 15 galaxies with disks and 9 with large amount of dust in filaments. Thirteen of the 24 dusty galaxies have a radio detection (54\%), while 4 out of 12 non-dusty galaxies show a detection $(33 \%)$. There is no significant difference in radio luminosity between the galaxies with disky and filamentary dust: $60 \%$ detections in galaxies with disks and $44 \%$ in galaxies with filaments. This finding is in general agreement with the findings by Tran et al. (2001).

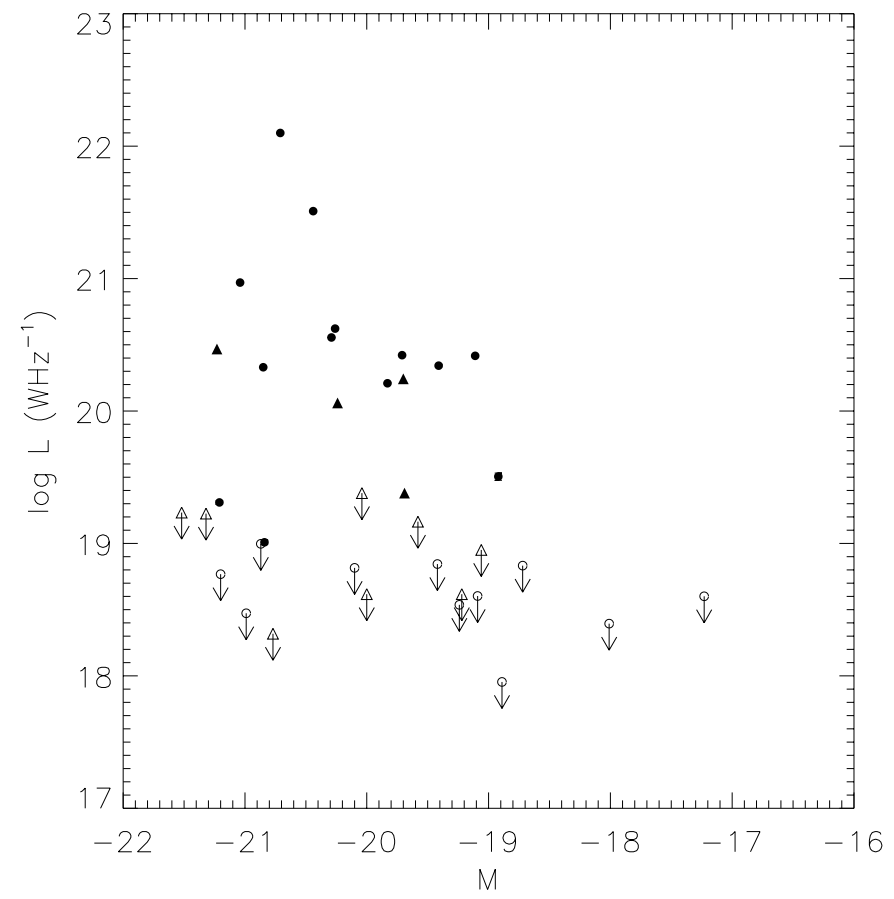

Fig. 3. Plot of log radio luminosity in $\mathrm{W} \mathrm{Hz}^{-1}$ versus absolute optical magnitude. Filled symbols are radio-detected nuclei of galaxies, while open symbols indicate upper limits for the rest of the galaxies. Triangles are sources in galaxies without dust and circles are sources in dusty galaxies. Sources in dusty galaxies have a slight tendency for being more powerful than the sources in non-dusty galaxies. Luminosity error bars are smaller than the symbols.

The relationship between optical absolute magnitude and radio luminosity for our weak radio sources is shown in Fig. 3. There is little difference in the distributions of the dusty and non-dusty galaxies, except perhaps that the three most powerful galaxies are all dusty. As expected, the more powerful radio sources are found in the brighter galaxies.

Most nearby high luminosity radio sources are found in dusty early type galaxies (de Koff et al. 2000), which suggests a link between dust and the existence of a radio source. Our wish now is to see if at the lower levels of radio luminosity dust also plays an important role. We divide the detections in two sets of sources: dusty and non-dusty according to the descriptions in Tran et al. (2001). As we see above, the dusty galaxies show a somewhat higher detection rate, but, given the steepness of the RLF, this could be influenced by slight differences in the distances to the two samples, or slight differences in the achieved sensitivities. Therefore it is more meaningful to compare the RLFs of the two samples than the detection percentages.

\subsection{Comparison of RLFs}

The integral RLFs for the two samples, computed by the same algorithm as that in Fig. 1 for the whole sample, are shown in Fig. 4. In this representation also, the dusty galaxies seem more active, but the difference is relatively small (a factor of $\sim 1.6$ ) and we wish to test the significance of this difference.

We have tried two statistical tests: Kolmogorov-Smirnoff (K-S) and a test using maximum likelihood method (ML). 
Table 2. Estimated integral luminosty coefficients. Values of coefficeints of estemated integral luminosity function $F$ obtained by maximum likelihood method. Errors are $1 \sigma$ estimates.

\begin{tabular}{ccccc}
\hline \hline data sample & $\alpha$ & $\beta$ & $x_{c}$ & $A_{0}$ \\
\hline all data & $-0.6 \pm 0.3$ & $0.80 \pm 0.10$ & $20.41 \pm 0.04$ & $0.21 \pm 0.04$ \\
dust data & $-0.6 \pm 0.4$ & $0.74 \pm 0.09$ & $20.40 \pm 0.10$ & $0.25 \pm 0.07$ \\
non-dust data & $-0.5 \pm 0.3$ & $1.20 \pm 0.60$ & $20.24 \pm 0.04$ & $0.14 \pm 0.02$ \\
\hline
\end{tabular}

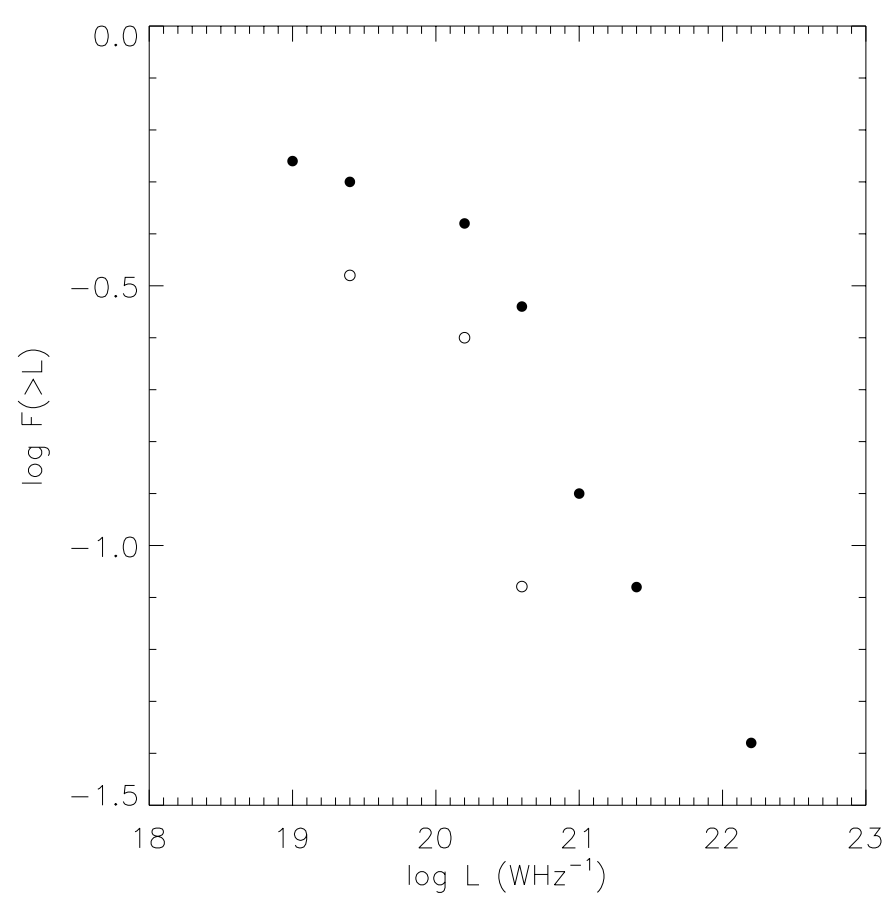

Fig. 4. The separated integral luminosity function. Open circles present the RLF for sources from galaxies lacking dust. Filled circles present the RLF of sources from dusty galaxies. Statistical tests show that the two distributions are not distinguishable, suggesting that dust is not important for the existence of low-luminosity AGN in nearby early-type galaxies.

The K-S test has the advantage of being parameter and form free, but the disadvantage of not being very conclusive for small samples. We have two data sets, one with 13 sources in dusty galaxies and one with 4 sources in non-dusty galaxies. We used routines from Numerical Recipes (Press et al. 1992). The probability that these two observation sets could be obtained from the same RLF is $64 \%$, hence the RLFs are statistically indistinguishable. However, the K-S test is sensitive to the effective number of data points, $N_{\mathrm{e}}$, which in our case of two distributions is $N_{\mathrm{e}}=N_{1} N_{2} /\left(N_{1}+N_{2}\right)=3.1$. Press et al. (1992) give $N_{\mathrm{e}} \geq 4$ as a limit for a decent accuracy. Thus, the above probability is not very accurate, but it still implies that the two data sets (two luminosity functions) are not significantly different.

Another approach that is more sensitive, but requires more a priori assumptions, is to fit a specific, parameterized, function to the RLF data using the maximum likelihood method, and compare the fitted functions. Since there is a limited number of degrees of freedom in the function, more powerful statistical statements can be made. To estimate the integral luminosity

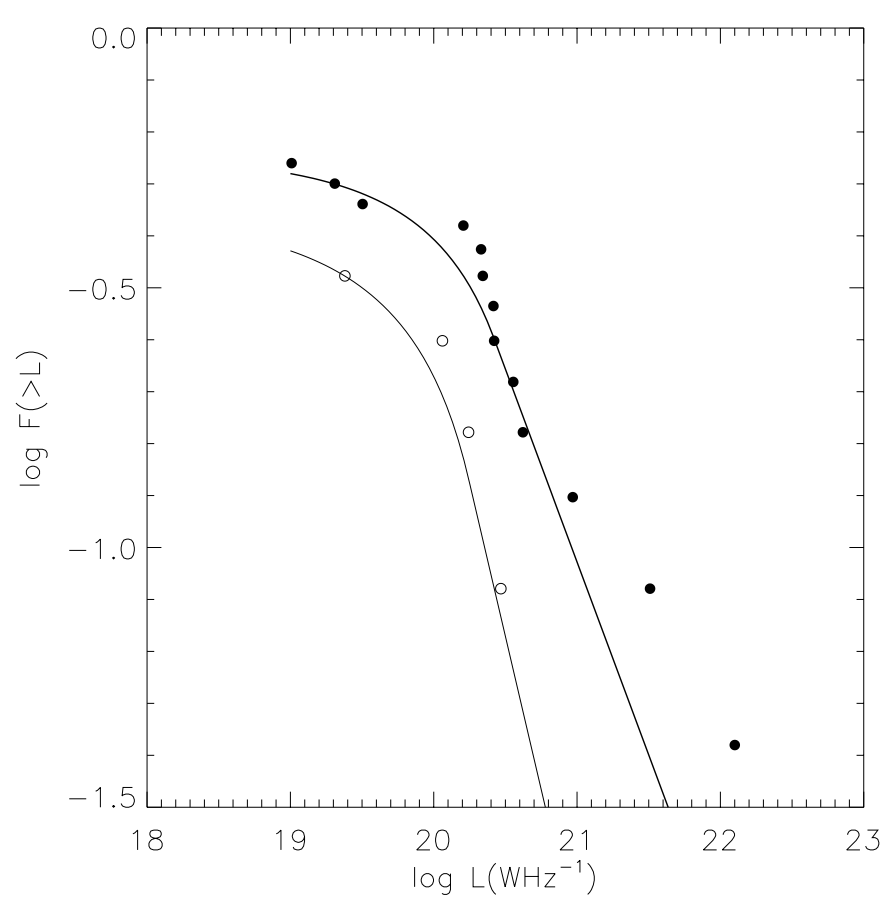

Fig. 5. Estimated integral luminosity functions compared to the data. The drawn lines show the ML model fits (Eqs. (2) and (3)) to the data, while the points are computed from individual detections. Field circles present dusty galaxies while open circles present non-dusty galaxies. The thick line shows the ML model fit for dusty galaxies and the thin line for non-dusty galaxies.

function we used a set of two power-law functions allowing for a break in the RLF. Our choice is similar to some previously used functions (Auriemma et al. 1977):

$\begin{array}{ll}F=A_{0} \times 10^{-\beta\left(x-x_{\mathrm{c}}\right)} & \text { for } x>x_{\mathrm{c}} \\ F=A_{0} \times\left(1+\frac{\beta}{\alpha}\left(10^{-\alpha\left(x-x_{\mathrm{c}}\right)}-1\right)\right) & \text { for } x<x_{\mathrm{c}}\end{array}$

where $x=\log _{10} L$, and $L$ is radio luminosity in $\mathrm{W} \mathrm{Hz}^{-1}$. The normalization constant $A_{0}$ is chosen so that at $x=x_{\mathrm{c}}, F=A_{0}$. Originally we assumed that at low luminosities the value of $F(x)$ had to approach $F=1$ as $x \rightarrow-\infty$, thus providing an additional constraint on the model. These solutions provided poor fits to the data and were dropped. This implies, however, that there is another break (or continuous change of slope) in the RLFs below the limits of our survey.

The system of coefficients $\alpha, \beta$ (slopes of the curve), $x_{\mathrm{c}}$ (the position of the break) and $A_{0}$ (normalization) that maximize the probability in the method, provide also the best fit to the data. Table 2 contains the calculated values for $\alpha, \beta, x_{\mathrm{c}}$ and $A_{0}$. 
The best-fit integral luminosity functions are compared to the observed values in Fig. 5. The symbols are filled circles for dusty and open circles for non-dusty sources. The thick line represents the model fit to dusty sources, while the thin line shows the fit to the non-dusty. The model curves fit the individual points reasonably well. The three radio brightest galaxies lie somewhat above the best two power-law fit in the region of the break, but the best fit value of the slope above the break, $\beta \simeq 0.75$, agrees with the slope measured by Sadler et al. (2002) (Fig. 2) based on much more data in the higher luminosity ranges.

The ML parameters $\alpha, \beta$, and $x_{\mathrm{c}}$ are essentially identical for dusty and non-dusty galaxies, indicating that the forms of the RLF are similar. Not surprisingly the normalization $A_{0}$ is higher for the dusty galaxies by a factor of about 1.8 , but this is only 1.6 times the uncertainty.

Another way to globally judge the significance of the difference between these RLFs it to ask if the "true" RLF were given by the dusty model, how unlikely is it that we would only detect four (or less) of the twelve non-dusty galaxies. If this probability is small, then the samples are significantly different. From Poisson statistics the probability of 4 or less non-dusty detections given the dusty RLF (thick line in Fig. 5 or the second line values in the Table 2) is $27 \%$, indicating a low statistical significance. If, hypothetically, the true non-dusty RLF is a factor of 1.6 lower than the dusty RLF we can ask how many non-dusty galaxies must be surveyed in order to demonstrate the RLF difference at a reliability of, say, $5 \%$. Repeating the Poisson analysis indicates that a sample about four times the current size is needed, or about 50 non-dusty galaxies.

Perhaps the most important result of this investigation is that in any case, a sizable fraction of the non-dusty galaxies, $\sim 30 \%$, are radio emitters, so that the presence of visible dust is not necessary for radio emission from an AGN.

\section{Conclusions}

We report $3.6 \mathrm{~cm}$ VLA observations of a sample of 36 nearby ellipticals selected on their optical/IR properties. We detected 17 unresolved (except the jet in NGC5322), compact, flat-spectrum radio cores associated with the central 1 " of the nuclei, suggesting that all detected sources are low luminosity AGNs. The lowest detected luminosities are $\sim 10^{19} \mathrm{~W} \mathrm{~Hz}^{-1}$.

We determine the Radio Luminosity Function (RLF) from these galaxies down to a luminosity almost two orders of magnitude lower in luminosity than previously published studies. It shows the continuation in the rise of space density of sources with AGN signature, which was expected from other, unpublished, studies (Condon, private communication). At the luminosities considered (i.e. $L \sim 10^{19}-10^{22} \mathrm{WHz}^{-1}$ ), the space densities of the AGNs and starburst galaxies approach each other, becoming hardly distinguishable. At the lower luminosity end of our sample $\sim 50 \%$ of E/S0 galaxies have detectable radio-AGNs.

Although the non-dusty galaxies show an indication of a lower probability of radio emission, the difference is not statistically significant in a sample of this size. Dust detectable in HST images is certainly not necessary for nuclear radio emission. This situation may be different for the more powerful radio galaxies observed in earlier surveys.

This takes us back to the question of fuel for the central engine of our low luminosity AGNs. If fuel is necessary for nuclear activity why do we find weak AGNs without visible dust? It should be noted that our non-dusty galaxies with radio detections lay further away $(D>30 \mathrm{Mpc})$ and it might be possible that small amounts of dust were not detected. Also, extremely diffuse gas and dust would not be visible (Goudfrooij \& de Jong 1995) but current theories of accretion require bars and disks and other distinct structures, so fueling from diffuse gas seems unlikely. Similarly, these galaxies could be fueled by hot, dust-free gas, but it seems unlikely that any mechanism in these low-luminosity sources would destroy dust more than in the high luminosity sources were dust is common. A more likely explanation is that the amount of dust and gas present near the nucleus is in some sense positively correlated with the AGN luminosity. The sources in our study are two to three orders of magnitude less luminous than the $3 \mathrm{C}$ sources in de Koff et al. (2000), where typical dust optical depths were of order unity. In the HST images, optical depths of less than $1 \%$ would probably be missed. Alternatively, AGN fueling may be cyclic, and AGN radio emission is now fueled from material at a few Schwarzschild radii, after the material in a larger circumnuclear accretion disk has been temporarily consumed.

The luminosity of an AGN is determined by the fueling rate and the mass-to-radiation conversion efficiency. The latter is influenced by the degree of advection which is in turn influenced by the Eddington luminosity and the mass of the $\mathrm{BH}$. As recent evidence suggests (Ho 2002 and references therein) many of the characteristics of low luminosity AGN could be explained by an advection-dominated accretion flow (Narayan \& Yi 1995; Narayan et al. 1998). The explanation of the dust/radio emission/luminosity relations may perhaps be found when we know the BH masses of the galaxies, or when we understand the characteristics of non-steady accretion flows.

Acknowledgements. The VLA is operated by the National Radio Astronomy Observatory for the U.S. National Science Foundation.

\section{References}

Auriemma, C., Perola, G. C., Ekers, R. D., et al. 1977, A\&A, 57, 41 Goudfrooij, P., Hansen L., Jørgensen, H. E., \& Nørgaard-Nielsen, H. U. 1994, A\&AS, 105, 341

Groudfrooij, P., \& de Jong, T. 1995, A\&A, 298, 784

Condon, J. J., Cotton, W. D., Greisen, E. W., et al. 1998, AJ, 115, 1693

Condon, J. J., \& Broderick, J. J. 1988, AJ, 96, 30

Condon, J. J., \& Broderick, J. J. 1991, AJ, 102, 1663

Condon, J. J. 1991, The Interpretation of Modern Synthesis Observations of Spiral Galaxies, ASP Conf. Ser., 18, 113

Condon, J. J., Huang, Z.-P., Yin, Q. F., \& Thuan, T. X. 1991, ApJ, 378, 65

Ferrari, F., Pastoriza, M. G., Macchetto, F., \& Caon, N. 1999, A\&AS, 136,269

Ho, L. C. 2002, ApJ, 564, 120

de Koff, S., Best, P., Baum, S. A., et al. 2000, ApJS, 129, 33

Narayan, R., Mahadevan, R., \& Quataert, E. 1998, Theory of Black Hole Accretion Disks, 148 
Narayan, R., \& Yi, I. 1995, ApJ, 452, 710

Osterbrock, D. E. 1989, Astrophysics of gaseous nebulae and active galactic nuclei, University Science Books

Perrett, K. M., Hanes, D. A., Butterworth, S. T., et al. 1997, AJ, 113, 895

Phillips, M. M., Jenkins, C. R., Dopita, M. A., Sadler, E. M., \& Binette, L. 1986, AJ, 92, 503

Press, W. H., Teukolsky, S. A., Vetterling, W. T., \& Flannery, B. P. 1992, Numerical recipes: The Art of Scientific Computing (CUP, Cambridge)

Rest, A., van den Bosch, F. C., Jaffe, W., et al. 2001, AJ, 121, 2431

Sadler, E. M., \& Gerhard, O. E. 1985, MNRAS, 214, 177
Sadler, E. M., Jenkins, C. R., \& Kotanyi, C. G. 1989, MNRAS, 240, 591

Sadler, E. M., Jackson, C. A., Cannon, R. D., et al. 2002, MNRAS, 329, 227

Tomita, A., Aoki, K., Watanabe, M., Takata, T., \& Ichikawa, S. 2000, AJ, 120,123

Tran, H. D., Tsvetanov, Z., Ford, H. C., et al. 2001, AJ, 121, 2928

van Dokkum, P. G., \& Franx, M. 1995, AJ, 110, 2027

Verdoes Kleijn, G. A., Baum, S. A., de Zeeuw, P. T., \& O’Dea, C. P. 1999, AJ, 118, 2592

Wrobel, J. M. 1991, AJ, 101, 127

Wrobel, J. M., \& Heeschen, D. S. 1991, AJ, 101, 148 